\title{
Densest Packings of Typical Convex Sets Are Not Lattice-Like*
}

\author{
G. Fejes Tóth
}

Mathematical Institute of the Hungarian Academy of Sciences, P.O. Box 127, Budapest H-1364, Hungary

gfejes@math-inst.hu

\begin{abstract}
We show that if $P$ is a convex polygon which has no parallel sides, then the densest packing of the plane with congruent copies of $P$ is not lattice-like. As a corollary we obtain that, in the sense of Baire categories, for most convex disks densest packing is not lattice-like.
\end{abstract}

\section{Introduction}

The packing density $\delta(C)$ of a set $C$ with finite positive Lebesgue measure is defined as the supremum of the densities of all packings of congruent copies of $C$. The lattice-packing density $\delta_{\mathrm{L}}(C)$ of $C$ is the supremum of the densities of all lattice packings of $C$. We prove the following:

Theorem 1. If $P$ is a convex polygon which has no parallel sides, then

$$
\delta_{L}(P)<\delta(P)
$$

Let $\mathscr{C}$ be the space of all convex disks in the plane equipped with the Hausdorff metric. It is obvious that the class $\mathscr{Q}$ of polygons which do not possess parallel sides is dense in $\mathscr{C}$. Denote by $\mathscr{N}$ the subset of $\mathscr{E}$ consisting of all disks $C$ for which

*This research was supported by the Hungarian National Foundation for Scientific Research (OTKA) under Grant Nos. 1907 and 14218. 
$\delta(C) \neq \delta_{\mathrm{L}}(C)$. It immediately follows from the continuity of the functionals $\delta(C)$ and $\delta_{\mathrm{L}}(C)$ that $\mathscr{N}$ is open in $\mathscr{E}$. Thus we obtain the following:

Theorem 2. Let $\mathscr{C}$ be the space of all convex disks in the plane equipped with the Hausdorff metric. Let $\mathscr{N}$ be the subset of $\mathscr{C}$ consisting of all convex disks $C$ for which $\delta(C)>\delta_{\mathrm{L}}(C)$. Then $\mathscr{N}$ is an open dense set in $\mathscr{C}$.

This means that, in the sense of Baire categories, for most convex disks no densest packing is lattice-like. An analogous theorem for covering has been proved in a joint paper of the author and Zamfirescu [1]. The first result establishing packing properties of typical convex sets is due to Gruber [3]. He showed that typical convex sets have surprisingly few neighbors in their densest lattice packing. For surveys on properties of typical convex sets in the Baire category sense we refer to [4] and [9].

The proof of Theorem 1 is given in Section 3. In Section 2 we prepare the proof of Theorem 1 by introducing generalized Dirichlet cells which give some insight into the structure of a lattice packing of a given convex disk $C$. We conclude the paper by mentioning some open problems in Section 4.

\section{Generalized Dirichlet Cells}

In this section we introduce and investigate generalized Dirichlet cells associated with the members of a lattice packing. Such cells were first introduced in [2] for packings of finitely many convex disks contained in a hexagon. For lattice packings the cells can be constructed similarly. We need, however, to describe the construction explicitly, in order to derive some important properties of these objects.

We proceed in two steps: First we consider centrally symmetric disks; the construction for general convex sets will be reduced to this case. Let $C^{*}$ be a centrally symmetric convex disk with center at the origin $o$ and let $\Lambda$ be a lattice such that the arrangement of disks $\mathscr{P}^{*}=\left\{C^{*}+x ; x \in \Lambda\right\}$ constitutes a packing. We start with choosing an appropriate base for $\Lambda$. To this end we make use of the distance function $f(x)$ of $C^{*}$ defined by

$$
f(x)=\sup _{\varrho x \in C^{*}} \varrho
$$

Let $a \in \Lambda, a \neq o$, be such that

$$
f(a) \leq f(x) \quad \text { for all } \quad x \in \Lambda
$$

Next we chose an element $b$ of $\Lambda$ linearly independent from $a$ such that $f(b) \leq f(x)$ for all $x \in \Lambda$ independent from $a$ and, furthermore, if $f(b)=f(a)$ we also assume that $\frac{1}{2}(a+b) \notin \Lambda$. We claim that the parallelogram $P$ with the vertices $o, a, a+b$, and $b$ is a fundamental region of the lattice $\Lambda$. To see this we consider the closed parallelogram $\tilde{P}$ with the vertices $a, b,-a$, and $-b$ and observe that for any point 
$x \in$ int $\tilde{P}$ we have $f(x)<f(b)$. Thus this parallelogram does not contain any lattice point other than $o$. Observing that

$$
\text { int } P \backslash\left\{\frac{1}{2}(a+b)\right\} \subset \operatorname{int} \tilde{P} \cup \operatorname{int}(\tilde{P}+a) \cup \operatorname{int}(\tilde{P}+a+b) \cup \operatorname{int}(\tilde{P}+b) \text {, }
$$

it easily follows that the only point of int $P$ which can be a point of $\Lambda$ is $\frac{1}{2}(a+b)$. This, however, was excluded by the choice of $b$. Thus $P$ is a fundamental parallelogram of $\Lambda$, and $a$ and $b$ form a base of $\Lambda$.

Observe that two disjoint translates of a centrally symmetric convex disk can be separated by a straight line passing through the midpoint of the segment connecting their centers. In particular, to any pair of points $x$ and $y$ from $\Lambda$ there is a line through $\frac{1}{2}(x+y)$ which separates $\frac{1}{2} f(x-y) C^{*}+x$ and $\frac{1}{2} f(x-y) C^{*}+y$. Let $L_{1}^{*}$ be a line through $\frac{1}{2} a$ separating $\frac{1}{2} f(a) C^{*}$ and $\frac{1}{2} f(a) C^{*}+a$. Consider the half-open parallel strip between the lines $L_{1}^{*}$ and $-L_{1}^{*}=L_{1}^{*}-a$ which contains the bounding line $L_{1}^{*}$ but not the line $-L_{1}^{*}$. This strip contains exactly one lattice point of the form $k a+b, k$ integer; let this well-defined point be denoted by $c$. Now we separate $\frac{1}{2} f(c) C^{*}$ and $\frac{1}{2} f(c) C^{*}+c$ by a line $L_{2}^{*}$ through $\frac{1}{2} c$, and, finally, we draw a line $L_{3}$ through $\frac{1}{2}(c-a)$ separating $\frac{1}{2} f(c-a) C^{*}$ from $\frac{1}{2} f(c-a) C^{*}+c-a$. Further, we introduce the lines $L_{4}^{*}=L_{1}^{*}-a=-L_{1}^{*}, L_{5}^{*}=L_{2}^{*}-c=-L_{2}^{*}$, and $L_{6}^{*}=L_{3}^{*}+a-c$ $=-L_{3}^{*}$. For $i=1, \ldots, 6$ let $H_{i}^{*}$ be the half-plane bounded by $L_{i}^{*}$ containing $C^{*}$. We assume that $a$ and $b$ are chosen so that $\frac{1}{2}(a+c) \notin$ int $H_{1}^{*} \cap H_{2}^{*}$. This can be achieved, if it is not already the case, by changing $a$ to $-a$.

We consider the set $D^{*}=\bigcap_{i=1}^{6} H_{i}^{*}$. It is obvious that $D^{*}$ contains $C^{*}$. We observe that $a$ and $c$ also form a base of $\Lambda$ and the parallelogram $\hat{p}$ with the vertices $o, a, c$, and $a+c$ is a fundamental region of $\Lambda$. Next we show that the intersection of $D^{*}$ with the angular region $\angle a o c$ is contained in $\hat{P}$.

In view of the assumption $\frac{1}{2}(a+c) \notin \operatorname{int}\left(H_{1}^{*} \cap H_{2}^{*}\right)$ we can distinguish the following two cases: $\frac{1}{2}(a+c) \notin$ int $H_{1}^{*}$ or $\frac{1}{2}(a+c) \notin$ int $H_{2}^{*}$. In the first case we observe that $f(a) \leq f(c)$ implies that $\frac{1}{2} a+c \notin H_{2}^{*}$, hence $D^{*} \cap \angle a o c$ is contained in the trapezoid with the vertices $o, \frac{1}{2} a, \frac{1}{2} a+c$, and $\frac{1}{2} c$, consequently also in $\hat{P}$. In the second case we use the assumption that $a+c \notin H_{1}^{*}$. It readily follows that in this case $D^{*} \cap \angle a O c$ is contained in the trapezoid with the vertices $o, \frac{1}{2} a, \frac{3}{4} a+\frac{1}{2} c$, and $\frac{1}{2} c$ which is again a subset of $\hat{P}$. A similar argument yields that $D^{*} \cap \angle(-a) o c \subset \hat{P}-a$. Using the central symmetry of $D^{*}$, it follows that

$$
D^{*} \subset \hat{P} \cup(\hat{P}-a) \cup(\hat{P}-a-c) \cup(\hat{P}-c) \text {. }
$$

As an immediate consequence we get that $D^{*}$ is bounded, hence it is a, possibly degenerate, centrally symmetric hexagon. In what follows if we speak of hexagons, we include degenerate cases, thus by a hexagon we mean a polygon with at most six sides. As a further consequence we mention that the collection of sets $\mathscr{D}^{*}=\left\{D^{*}+\right.$ $x ; x \in \Lambda$ \} forms a packing. In order to see this we have to show that the sets $\hat{P} \cap \operatorname{int}\left(D^{*}+x\right), x \in \Lambda$, constitute a packing. In view of the last relation only four of these sets are nonempty, namely, the sets $\hat{P} \cap \operatorname{int} D^{*}, \hat{P} \cap \operatorname{int}\left(D^{*}+a\right)$, $\hat{P} \cap \operatorname{int}\left(D^{*}+c\right)$, and $\hat{P} \cap \operatorname{int}\left(D^{*}+a+c\right)$ and it is easy to check that they are mutually disjoint. 
The sets $D^{*}+x, x \in \Lambda$, are called generalized Dirichlet cells associated with $\mathscr{P}^{*}$ (Fig. 1). It should be observed that the construction of the generalized Dirichlet cells is, in general, not unique. Although it can be shown that in the case of a densest packing the construction is unique, we do not make use of this fact. We also note that these cells do not necessarily cover the plane. However, in the case when the generalized Dirichlet cells leave a portion of the plane uncovered, the cells, which are centrally symmetric hexagons, can be rearranged to form a lattice tiling. The corresponding copies of $C^{*}$ form a lattice packing with higher density than the original arrangement. This implies that:

(i) For a densest packing, that is, when $\mathscr{P}^{*}$ has density $\delta_{\mathrm{L}}\left(C^{*}\right)$, the generalized Dirichlet cells form a tiling. Moreover, in this case $D^{*}$ has the minimum area among all centrally symmetric hexagons containing $C$.

Now let $C$ be an arbitrary, not necessarily centrally symmetric, convex disk and let $\Lambda$ be a lattice such that $\mathscr{P}=\{C+x ; x \in \Lambda\}$ is a packing. Consider the set $C^{*}$ $=\frac{1}{2}(C-C)$. Note that $C^{*}$ is centrally symmetric with center $o$. We recall the well-known observation of Minkowski [6] that $\mathscr{P}$ is a packing if and only if the corresponding arrangement $\mathscr{D}^{*}=\left\{C^{*}+x ; x \in \Lambda\right\}$ is a packing. This follows immediately from the fact that the sets $C+x$ and $C+y$ can be separated by a straight line of given direction if and only if $C^{*}+x$ and $C^{*}+y$ can be separated by lines of the same direction. We consider the generalized Dirichlet cells to the packing $\mathscr{P}^{*}$ as constructed above, and observe that $C$ can be separated from $C+a, C+c$, and $C+a-c$ by straight lines $L_{1}, L_{2}$, and $L_{3}$ parallel to $L_{1}^{*}, L_{2}^{*}$, and $L_{3}^{*}$, respectively. We introduce the lines $L_{4}=L_{1}-a, L_{5}=L_{2}-c$, and $L_{6}=L_{3}-a+c$ and consider, for $i=1, \ldots, 6$, and half-plane $H_{i}$ bounded by $L_{i}$ and containing $C$. The generalized Dirichlet cell $D$ associated with $C$ is defined as $D=\cap_{i=1}^{6} H_{i}$ (Fig. 2). Obviously, $C \subset D$. Furthermore, since $D$ is the intersection of three parallel strips and $2 D^{*}$ is nothing else but the intersection of the difference sets of these strips, we have $\frac{1}{2}(D-D) \subset D^{*}$. Since the collection of the sets $\mathscr{D}^{*}=\left\{D^{*}+x ; x \in \Lambda\right\}$ is a packing, so is the collection $\mathscr{D}=\{D+x ; x \in \Lambda\}$.

Define the sets $Q$ and $Z$ by

$$
Q=H_{2} \cap H_{3} \cap H_{5} \cap H_{6}
$$

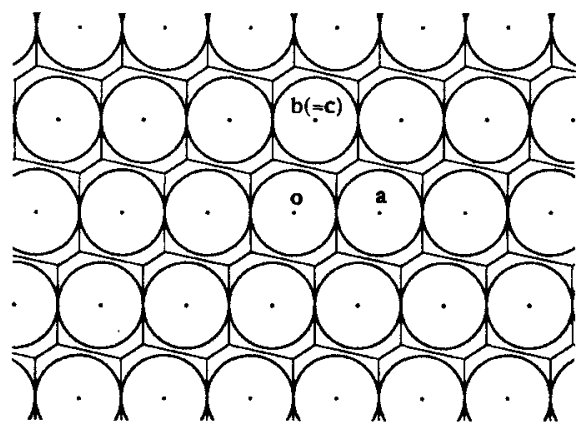

Fig. 1.

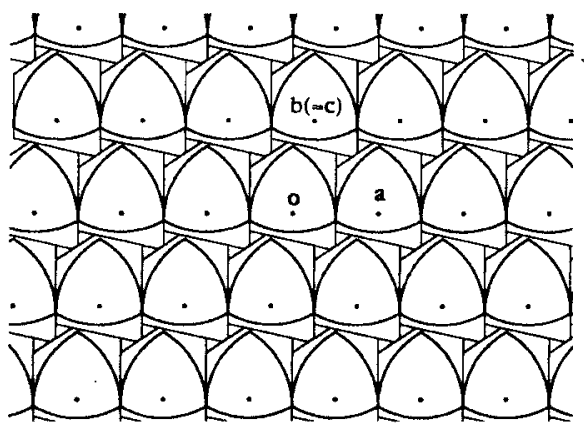

Fig. 2. 


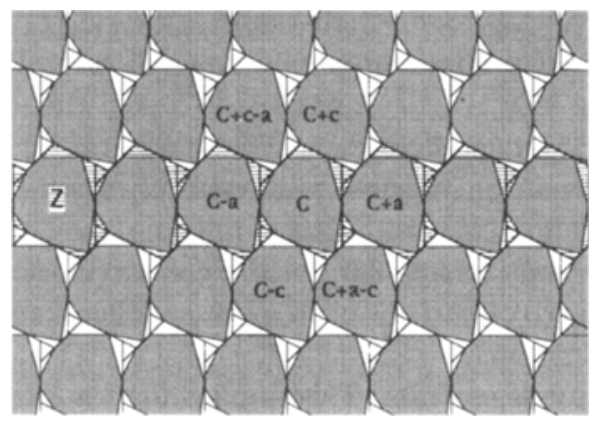

Fig. 3.

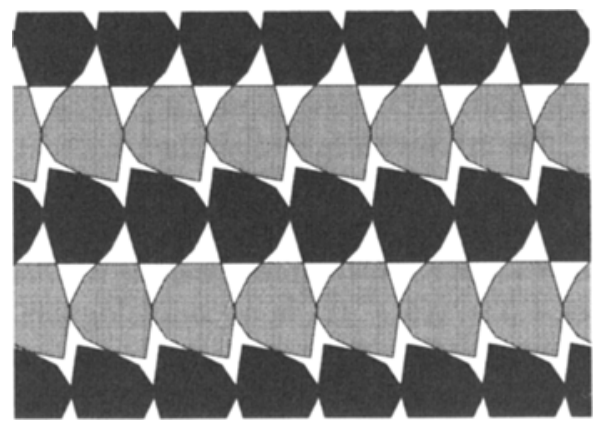

Fig. 4.

and

$$
Z=\bigcup_{i=-\infty}^{\infty}(Q+i a)
$$

Obviously, $Z$ is centrally symmetric, it contains all the sets $C+i a, i=0, \pm 1, \pm$ $2, \ldots$, and all other members of $\mathscr{P}$ are disjoint from $Z$ (Fig. 3). Let $d$ be the center of symmetry of $Q$. Then the sets $-C+i a+2 d, i=0, \pm 1, \pm 2, \ldots$, are contained in $Z$, therefore we can replace the sets $C+i a, i=0, \pm 1, \pm 2, \ldots$, of $\mathscr{P}$ by these sets so that the new arrangement still constitutes a packing. Any other row of $\mathscr{P}$ can be flipped over in a similar way. In particular, we can replace the disks $C+i a+2 j c$, by the disks $-C+i a+2 d+2 j c, i, j=0, \pm 1, \ldots$ (Fig. 4). Thus we obtained the following:

Lemma. Let $C$ be a convex disk and let $\Lambda$ be a lattice such that $\mathscr{P}=\{C+x ; x \in \Lambda\}$ is a packing. Then a base $\{a, c\}$ of $\Lambda$ and a vector $d$ exist such that the system of disks

$$
\begin{array}{r}
-C+i a+2 d+2 j c \\
C+i a+(2 j+1) d
\end{array} \quad(i, j=0, \pm 1, \pm 2, \ldots)
$$

is a packing.

\section{Proof of Theorem 1}

Let $C \in \mathscr{Q}$ and let $\Lambda$ be a lattice such that $\mathscr{P}=\{C+x ; x \in \Lambda\}$ is a packing with density $d(\mathscr{P})=\delta_{\mathrm{L}}(C)$. We construct a nonlattice packing of congruent copies of $C$ with density exceeding $\delta_{\mathrm{L}}(C)$. Consider the packing $\mathscr{P}^{*}=\left\{C^{*}+x ; x \in \Lambda\right\}$, and construct generalized Dirichlet cells to $\mathscr{P}^{*}$ and $\mathscr{P}$, respectively, as described in Section 2. We preserve the notation introduced in Section 2. In view of Minkowski's observation mentioned above, $d(\mathscr{P})$ is related to the density $d\left(\mathscr{P}^{*}\right)$ of $\mathscr{P}^{*}$ by the formula

$$
d\left(\mathscr{P}^{*}\right)=d(\mathscr{P}) \frac{\operatorname{area}\left(C^{*}\right)}{\operatorname{area}(C)}
$$


Therefore, since $\mathscr{P}$ has maximal lattice density, so does $\mathscr{P}^{*}$, that is, $d\left(\mathscr{P}^{*}\right)=\delta_{\mathrm{L}}\left(C^{*}\right)$. It follows by (i) that $\mathscr{P}^{*}$ is a tiling and $D^{*}$ is of minimum area among all centrally symmetric hexagons containing $C^{*}$.

We start with excluding the case that $D^{*}$ is a parallelogram. We note that if for a centrally symmetric convex disk the minimum-area circumscribed centrally symmetric hexagon is a parallelogram, then the disk coincides with that parallelogram. Thus if $D^{*}$ is a parallelogram, then so is $C^{*}$. It is easy to see that, for the convex polygon $C, C^{*}=\frac{1}{2}(C-C)$ is a parallelogram if and only if $C$ itself is a parallelogram. However, $C \in \mathscr{Q}$, so it cannot be a parallelogram.

In what follows we assume that $D^{*}$ is nondegenerate. This implies that at most one of the lines $L_{2}$ and $L_{3}$ is parallel to $a$. Without loss of generality we assume that

(ii) $L_{2}$ and $a$ are not parallel.

Consider the pairs of opposite lines $L_{1}$ and $L_{6}$. Since $C \in \mathscr{Q}$, at least one of these lines intersects $C$ in a single point or in the empty set. Again, it will not restrict the generality if we assume that

(iii) $\operatorname{card}\left(C \cap L_{6}\right) \leq 1$.

Consider the packing consisting of translates of $C$ and $-C$ introduced in the lemma. Inequality (iii) implies that card $((-C+2 d) \cap(C+c-a)) \leq 1$. We investigate first the case when $(-C+2 d) \cap(C+c-a)=\varnothing$. Let $m$ be the minimum distance between points of $-C+2 d$ and $C+c-a$. In view of (ii) we can choose a vector $\tilde{c}$ such that $c-\tilde{c}$ is parallel to $L_{2},|c-\tilde{c}| \leq m$ and, furthermore, $a$ and $\tilde{c}$ span a parallelogram of smaller area than $a$ and $c$ do. Now, the system $\tilde{\mathscr{P}}$ of disks

$$
\begin{gathered}
-C+2 d+i a+j(c+\tilde{c}) \\
C+i a+\tilde{c}+j(c+\tilde{c})
\end{gathered} \quad(i, j=0, \pm 1, \pm 2, \ldots)
$$

is a packing with density greater than $d(\mathscr{P})=\delta_{\mathrm{L}}(C)$. For later reference we observe that the increment of density is of order of magnitude $|c-\tilde{c}|$. We also note that in this construction we did not make use of the supposition that the density of $\mathscr{P}$ is maximal.

If $(-C+2 d) \cap(C+c-a) \neq \varnothing$, then the construction above cannot be applied directly. However, we can proceed as follows. We introduce the vectors $a^{\prime}$ and $c^{\prime}$ so that $a-a^{\prime}$ is parallel to $L_{1}, c-c^{\prime}$ is parallel to $L_{2}, a-a^{\prime}-c+c^{\prime}$ is parallel to $L_{3}$, and let $\Lambda^{\prime}$ be the lattice generated by $a^{\prime}$ and $c^{\prime}$. We consider the arrangement of hexagons $\left\{D^{*}+x ; x \in \Lambda^{\prime}\right\}$ and observe that it arises from the tiling $\left\{D^{*}+x ; x \in \Lambda\right\}$ by sliding adjacent hexagons along the line of the common side. The new arrangement still forms a packing in which, if $\left|a-a^{\prime}\right|$ is sufficiently small, triangular gaps of sides $\left|a-a^{\prime}\right|,\left|c-c^{\prime}\right|$, and $\left|a-a^{\prime}-c+c^{\prime}\right|$ arise. The area of the triangular gaps is of magnitude $\left|a-a^{\prime}\right|^{2}$. Consequently, the density of the hexagons is of magnitude $1-\left|a-a^{\prime}\right|^{2}$. According to the observation of Minkowski mentioned above the system of hexagons $\left\{D+x ; x \in \Lambda^{\prime}\right\}$ also forms a packing, hence so does 
the corresponding system $\mathscr{P}^{\prime}=\left\{C+x ; x \in \Lambda^{\prime}\right\}$ of translates of $C$. Now we have $(-C+2 d) \cap\left(C+c^{\prime}-a^{\prime}\right)=\varnothing$ and, moreover, the minimum distance between points of $-C+2 d$ and $C+c^{\prime}-a^{\prime}$ is of magnitude $\left|a-a^{\prime}\right|$. Thus we can apply the construction above to $\mathscr{P}^{\prime}$ obtaining thereby a new packing $\tilde{\mathscr{P}}$. The decrement of density while we change from $\mathscr{P}$ to $\mathscr{P}^{\prime}$ is of magnitude $\left|a-a^{\prime}\right|^{2}$. On the other hand, as we pointed out above, the density can increase in the order $\left|a-a^{\prime}\right|$ when we change from $\mathscr{P}^{\prime}$ to $\tilde{\mathscr{P}}$. Thus, choosing $\left|a-a^{\prime}\right|$ sufficiently small, we can achieve that $d(\mathscr{\mathscr { P }})>d(\mathscr{P})$.

\section{Some Open Problems}

Our construction yields a nonlattice packing which is still very regular: namely, it is the union of two lattice packings. We say that an arrangement $\mathscr{A}$ of congruent copies of a body $C$ is $k$-periodic if a lattice $\Lambda$ and $k$ congruent copies $C_{1}, \ldots, C_{k}$ of $C$ exist such that $\mathscr{A}=\left\{C_{i}+x ; i=1, \ldots, k, x \in \Lambda\right\}$. Let $\delta^{(k)}(C)$ be the supremum of the densities of all $k$-periodic packings of $C$. Of course, a 1-periodic arrangement is just lattice-like, so we have $\delta_{\mathrm{L}}(C)=\delta^{(1)}(C)$. With this terminology Theorem 2 says that, for most convex disks $C, \delta^{(2)}(C)>\delta^{(1)}(C)$. Let $\mathscr{P}^{(k)}$ denote the subset of $\mathscr{C}$ consisting of all convex disks $C$ for which $\delta(C)=\delta^{(k)}(C)$ but $\delta(C)>\delta^{(i)}(C)$ for all $i<k$. Further, let $\mathscr{P}^{(\infty)}=\mathscr{C} \backslash \bigcup_{k=1}^{\infty} \mathscr{P}^{(k)}$. What can be said about the topological structure of these sets? Unfortunately, our knowledge concerning this question is very limited. Zamfirescu observed that some examples of pentagonal tiles (see pp. 492-497 of [5]) show that $\mathscr{P}^{(k)} \neq \varnothing$ for $k=2,3,4,6$, and 8 , however, we do not know whether $\mathscr{P}^{(k)}$ is empty or not for other values of $k$. Of particular interest is the question whether a convex disk in $\mathscr{P}^{(\infty)}$ exists.

It can be expected that a statement analogous to Theorem 2 holds in any dimension. Rogers [7, p. 15] conjectures that for sufficiently large dimensions even the densest packing of balls is not lattice-like. One of the main tools to the proof of Theorem 1 is the lemma stating that in a lattice packing of convex disks one can find a sublattice such that the image of this sublattice under a central reflection is disjoint from all other members of the packing. Can this statement be generalized to higher dimensions? Of course, sublattices of different dimension can be considered. It seems that if the lemma can be generalized at all to $E^{d}$, the easiest case is to show the existence of a layer $((d-1)$-dimensional sublattice) which can be flipped over.

Instead of the space $\mathscr{E}$ we could investigate the space $\mathscr{S}$ of all star-shaped bodies, which is also a Baire space with the Hausdorff metric. It seems that the occurrence of sets $S$ with $\delta(S)=\delta_{\mathrm{L}}(S)$, or generally with $\delta(S)=\delta^{(k)}(S)$, is even more sporadic in $\mathscr{S}$ than it is in $\mathscr{E}$. On the other hand, Schmitt [8] constructed star-shaped sets for which the densest packing cannot be realized by a periodic arrangement. Moreover, as Schmitt pointed out, taking a tiling by $k$ distinct hexagons, arranged in periodically repeated rows as starting point in his construction the existence of a star-shaped set $S$ with $\delta(S)=\delta^{(k)}(S)$ for arbitrary $k$ also follows.

Of course, all the problems mentioned here can be raised for coverings as well. As we mentioned in the Introduction, a theorem for covering analogous to 
Theorem 2 has been proved by Zamfirescu and the author. Our knowledge about these types of problems for packings and for coverings shows a complete analogy in all other respects.

\section{Acknowledgment}

I am indebted to Peter Schmitt for valuable remarks and suggestions.

\section{References}

1. G. Fejes Tóth and T. Zamfirescu, For most convex dises thinnest covering is not lattice-like, Intuitive Geometry, Colloquia Mathematica Societates János Bolyai, vol. 63, North-Holland, Amsterdam, 1994, pp. 105-108.

2. L. Fejes Tóth, Some packing and covering theorems, Acta Sci. Math. (Szeged) 12A (1950), 62-67.

3. P. M. Gruber, Typical convex bodies have surprisingly few neighbours in densest lattice packings, Studia Sci. Math. Hungar. 21 (1986), 163-173.

4. P. M. Gruber, Baire categories in convexity, Handbook of Convex Geometry (P. M. Gruber and J. M. Wills, eds.), North-Holland, Amsterdam, 1993, pp. 1327-1346.

5. B. Grünbaum and G. C. Shephard, Tilings and Patterns, Freeman, San Francisco, 1987.

6. H. Minkowski, Dichteste gitterförmige Lagerung kongruenter Körper, Nachr. Ges. Wiss. Göttingen (1904), 311-355 = Gesammelte Abhandlungen, vol. 2, Teubner, Leipzig, 1911, pp. 3-42.

7. C. A. Rogers, Packing and Covering, Cambridge University Press, Cambridge, 1964.

8. P. Schmitt, Disks with special properties of densest packing, Discrete Comput. Geom. 6 (1991), 181-190.

9. T. Zamfirescu, Baire categories in convexity, Atti Semin. Mat. Fis. Univ. Modena 39 (1991), 139-164.

Received October 1, 1993, and in revised form October 6, 1994. 